\title{
Urgences
}

\section{Le navet bleu}

\section{Jean-Marc Cormier}

Numéro 10, 2e trimestre 1984

Spécial fantasmes

URI : https://id.erudit.org/iderudit/025156ar

DOI : https://doi.org/10.7202/025156ar

Aller au sommaire du numéro

Éditeur(s)

Urgences

ISSN

0226-9554 (imprimé)

1927-3924 (numérique)

Découvrir la revue

Citer ce document

Cormier, J.-M. (1984). Le navet bleu. Urgences, (10), 47-64.

https://doi.org/10.7202/025156ar

Ce document est protégé par la loi sur le droit d'auteur. L'utilisation des services d'Érudit (y compris la reproduction) est assujettie à sa politique d'utilisation que vous pouvez consulter en ligne.

https://apropos.erudit.org/fr/usagers/politique-dutilisation/
Cet article est diffusé et préservé par Érudit.

Érudit est un consortium interuniversitaire sans but lucratif composé de l’Université de Montréal, l'Université Laval et l'Université du Québec à Montréal. Il a pour mission la promotion et la valorisation de la recherche. https://www.erudit.org/fr/ 


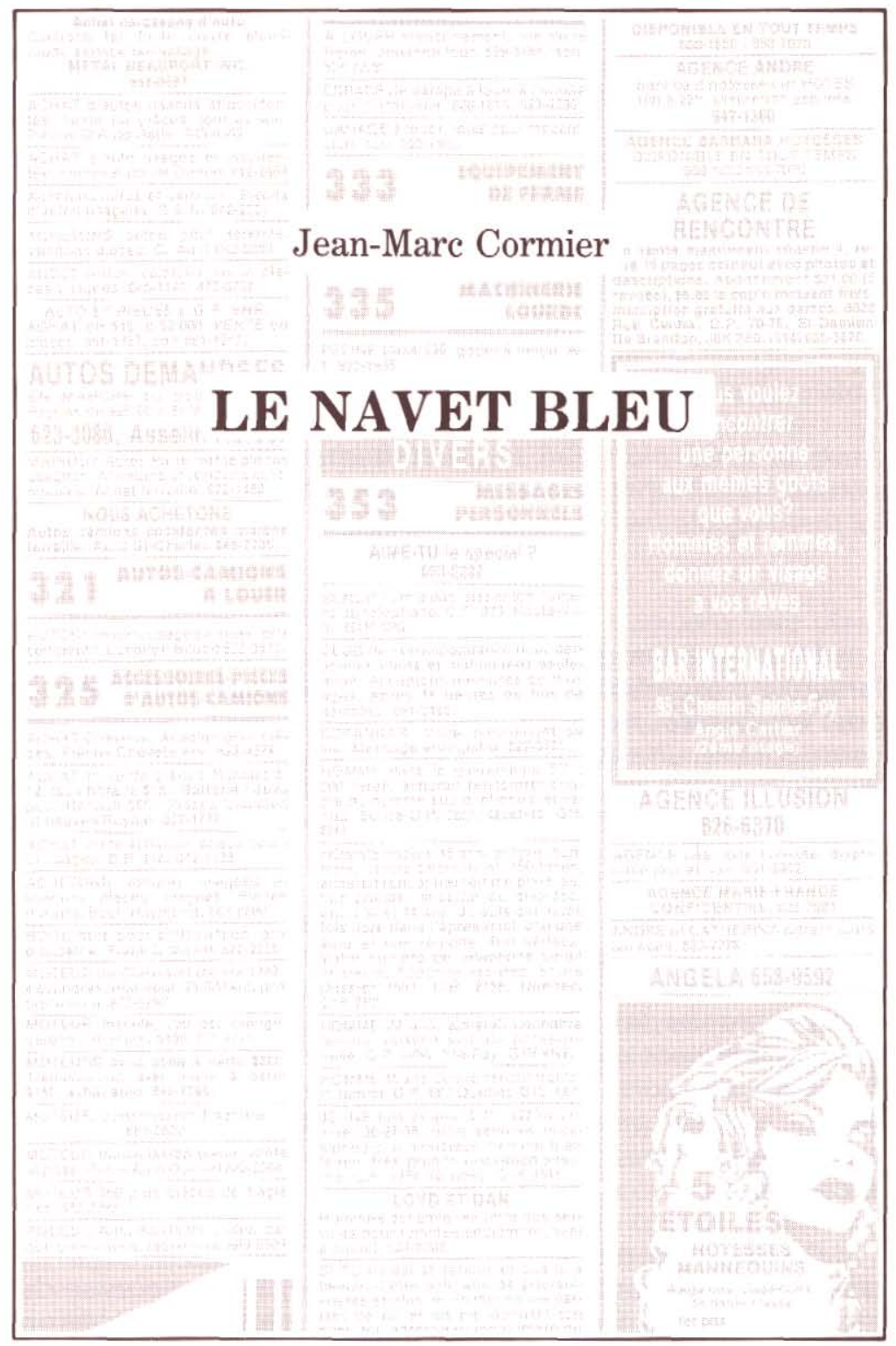


Il était un petit navire il était un petit navire qui n'avait ja ja jamais navigué qui n'avait ja ja jamais navigué

Nous jouions dans le pacage, Josephat Robert et moi, lorsqu'une espèce de mastodonte au nez cassé, aux cheveux drus coupés courts et à l'oeil morne, s'avança vers nous le sourcil grave, une esquisse de sourire sournois fichée sur des lèvres charnues qui laissaient à peine entrevoir deux rangées de dents jaunes passablement mal cordées et percées ça et là de petits orifices noirs.

Nous n'en doutions aucunement, il fallait fuir. II s'agissait d'une brute épaisse à la cervelle d'oiseau. II est, disons-le carrément, de ces expressions consacrées qui, quand on les utilise à propos de certains individus particulièrement grotesques, constituent une insulte à l'endroit des animaux qu'elles évoquent, une sorte de blasphème. Les besoins de l'oiseau en fait de matière grise sont certainement limités grâce à sa nature intrinsèque, d'une si pure limpidité et d'une si absolue simplicité.

Qu'une telle masse de débilité cartilagineuse, toute en crasseuse ignorance, toute en muscles et si peu pourvue de matière pensante fût en même temps construite comme un 
vicieux bulldozer avait de quoi inquiéter les petits garçons que nous étions. D'autant plus qu'elle s'avançait lentement, sûre d'elle-même, nous reluquant de son gros oeil, toute en sueurs, avec une généreuse broue blanche qui lui coulait maintenant sur le menton.

Et le lieu même de nos ébats qui avait lui-même subitement changé d'apparence. Autour de nous, de frêles épinettes brûlées jusqu'à mi-hauteur, la tête cassée pour la plupart. Sous nos pieds nus, le sol rocailleux couvert par-ci par-là d'une avare mousse de lichen. Et cette roulure de ciel gris-noir qui commençait à déverser sur nos corps une pluie glaciale.

II fallait fuir, bien sûr! II aurait fallu fuir. Mais la peur réfrigérante nous avait figés là. Nos jeunes corps étaient seulement parcourus de longs frissons qui couraient allègrement le long de nos échines.

Nous connaissions cette salope. Nous savions que Vieil Bidoune (c'était là le surnom que tous lui connaissaient) prenait un plaisir pervers à pincer de ses ongles durs les scrotums des petits garçons qu'il réussissait à attraper. Les tenant fermement entre ses jambes crochues, il finissait immanquablement, quand il avait assez joui à torturer leurs petits sacs, par leur cracher au cul de gros morviats verdâtres.

Sans même me retourner, les mains derrière le dos, la gorge nouée, je tâtonnai pour trouver le bouton, dans I'angle de la cuisse de la statue. Je l'atteignis enfin et, sentant se mouvoir souplement le bronze immense dans le cadrage de la porte (que faisait là ce truc bizarre?) j'attrapai prestement Josephat Robert par le poignet et le tirai violemment hors de ce lieu sordide et oppressant.

Complice de notre fuite, le bronze se remit en place d'un coup sec. II ne céderait certainement pas aux coups rageurs de la brute. II ne resta pas même une infime blessure dans le paysage. Nous étions de retour dans le pâturage accueillant. La vache meugla bonnement. Quelques hirondelles volaient dans le très beau ciel bleu, scrutant le sol, en quête de nourri- 
ture pour leurs petits. Cette journée de fin de mois d'août avait une odeur vaguement sucrée, douce, veloutée. Un calme absolu régnait sur la campagne.

Essoufflés encore, nous nous étions assis dans l'herbe. Nos rires gamins avaient vite chassé de nos esprits l'image horrifiante du monstre. Ce cauchemar en entier fut bientôt complètement oublié.

Josepha Robert avait mon âge. Il était de même taille, de même couleur, de même nature que moi. II m'accompagnait toujours dans mes jeux. J'avais besoin de lui, il m'était indispensable et je lui étais nécessaire. Nous étions faits pour être ensemble. Cela était une vérité si fondamentale qu'elle s'était cramponnée à nos fibres dès la naissance.

Je pris sa petite main dans ma petite main et nous partîmes, tout contents, tout joyeux, chantant à l'unisson une mélodie apprise dans un cahier de La Bonne Chanson. Nous avions surmonté la première épreuve. Nous étions toujours unis. Josephat Robert prenait soin de moi, je prenais soin de lui.

$$
\text { ***** }
$$

Au bout de cinq ou six semaines au bout de cinq ou six semaines les vivres vin vin vinrent à manquer les vivres vin vin vinrent à manquer

Décrire par le menu détail tout ce qui suivit constituerait une entreprise purement titanesque. J'essayerai seulement d'esquisser à larges traits, comme à coups de spatule, un tableau autant que faire se peut fidèle à tout le moins aux grandes lignes de notre aventure.

II était extrêmement difficile pour les petits enfants que nous étions de maintenir plausible la trame de la réalité, qui coulait autour de nous, nous enveloppant comme les acteurs 
d'un scénario abscons. Nous n'avions pas de pouvoir. Nous ne pouvions que nous observer jouer le jeu.

Toujours est-il qu'après une pause très brève, notre capacité de récupération étant phénoménale, nous avions repris nos jeux. Nous sautillions gaiement, nous tenant toujours par la main, comme siamois, comme nés ainsi, accrochés l'un à l'autre par une pièce de chair commune. Nos pas nous menaient vers la source. J'aimais en effet croire que cette mare odorante où nous pêchions les queues de poêlone était LA SOURCE. Nous disions à vrai dire, dans notre vocabulaire coloré des Hauts-de-la-Beauce, la ressource, prononcer larsourc'. Cela me semblait d'une divine musicalité. J'aimais donc follement patauger dans larsourc' et je lisais dans les yeux de Josephat Robert le même amour pur et clair pour cette mare beige où s'enfonçaient nos jambes jusqu'à mi-mollet dans la vase douce et chaude.

Mais nous n'y étions pas encore que tout le décor s'était de nouveau transformé tout autour de nous. Non seulement les meuglements rassurants de la Caille s'étaient-ils tus depuis un siècle déjà, mais le pacage tout entier avait cédé la place à un long corridor gris qui faisait glisser son épais tapis clouté sous nos petits pieds nus, guidant lui-même nos pas de manière si subtile que, même mus par une peur atroce, nous n'arrivions, malgré tous nos efforts, qu'à nous maintenir en place.

Nous avions peur de nouveau et nous avions mal. Nous n'avions rien à nous dire pour nous communiquer ces sensations, elles se lisaient sur nos corps, mais nos boîtes à mots dévidaient par elles-mêmes des flots automatiques et continus d'interjections et de demi-jurons.

D'abord il n'y eut rien d'autre que ce long corridor aux très hauts murs suintants qui ne nous laissaient entrevoir qu'une très fine raie de lumière d'un bleu glacé, loin, si terriblement loin, au-dessus de nos têtes. Déjà à bout de souffle, nous nous tenions serrés l'un contre l'autre et nous n'avancions plus qu'à tout petits pas feutrés, ce qui revient à dire que, désormais, 
nous n'avancions plus du tout, que le tapis gagnait même du terrain sur nous, que nous reculions.

II y avait aussi ce bruit. Ce frottement épais qui nous sembla monter du sol, produit par le tapis qui flottait apparemment sur de grosses billes parsemées sur une surface de métal.

Le plus vif de la douleur qui nous habitait se concentrait à la hauteur du plexus solaire et chacun devait garder une main légèrement appuyée sur sa poitrine comme pour empêcher le sang de s'échapper d'une blessure, comme pour prévenir une perforation définitive par l'intérieur.

Nous étions nus dans cette cage opaque. Parfaitement seuls et flambant nus avec l'impression lourde de porter sur nos frêles épaules le poids massif de I'histoire du monde. II n'était simplement pas possible pour nous de comprendre le sens de ce message. Heureusement, chacun pouvait s'appuyer sur l'autre pour mieux supporter cette anxiété monocorde qui semblait vouloir durer indéfiniment.

À un certain moment, le regard hébété de Josephat Robert me fit peur et je crois bien que le mien lui fit peur aussi. C'était comme si nos yeux étaient envahis à leur tour par l'étincelle sadique qui allumait l'oeil par ailleurs morne de Vieil Bidoune, le monstre rencontré à la première épreuve.

C'est alors que l'air ambiant devint soudainement sirupeux et glacial. C'était, chaque fois que nous aspirions, comme si un liquide gras et froid nous coulait dans les poumons. De grosses gouttes de brume liquéfiée par la froidure descendaient maintenant le long des murs, laissant derrière elles des sillons roses dans la crasse.

De multiples chuchotements surgirent soudainement de partout tout autour de nous. Des chuchotements qui nous semblaient palpables. Ou plutôt, des murmures matérialisés qui entreprenaient de nous palper. Et chacun de ces attouchements morbides laissait une sorte de brûlure sur nos petits 
corps transis. C'était la panique absolue. Nous avions peur et nous avions mal. Des crampes de fer nous tordaient les entrailles. Un liquide brun épais nous coulait le long des jambes. Le flux. II y eut ce moment plus douloureux encore où notre étreinte craintive sembla vouloir se défaire d'elle-même, comme cassée par nos tensions. Ce fut comme si tout allait finir à l'instant, et l'angoisse et la vie. Comme si chacun de nous devait mourir en se détachant de l'autre. Mais, sujette à un rythme qui nous échappait, dont nous n'avions plus le contrôle, elle se resserra bientôt plus vivement encore.

C'est à ce moment précis qu'apparut de nouveau Vieil Bidoune, comme surgi du néant, et sous une forme plus abominable encore. Un désir fou avait crispé tous ses muscles. II n'était plus qu'une boule de chair purulente avec une gueule avaleuse en son centre et qui roulait vers nous à toute vitesse.

Un même mouvement de frayeur nous secoua tous les deux de la tête aux talons. Une nanoseconde nous vit parfaitement emboîtés l'un dans l'autre, exactement superposés. Nous ne faisions plus qu'un dans la terreur. Puis l'un de nous, je ne sais plus lequel, se dégagea, mais continua de tendre la main vers l'autre. Nos mains parvinrent finalement à se saisir et s'agrippèrent frénétiquement I'une à I'autre. Mouillées de sueur mais soudées, formant une espèce de figure arthritique, nos mains d'enfants s'étaient décharnées jusqu'aux os et, dans la course folle que nous avions subitement, bien qu'inconsciemment entreprise, nous y jetions de temps à autre un regard d'angoisse et de muette stupéfaction.

Et nos pas! Et nos pas qui décidaient pour nous! Nos pas qui nous menaient à fond de train vers Vieil Bidoune qui, dans son immensité macabre, ouvrait la bouche et se pourléchait les babines, se préparant à nous avaler tout entiers.

Nos jambes étaient maîtresses de nous désormais. Nos jambes étaient vendues. Nous étions les esclaves de notre course éperdue et une rage totale nous animait corps et âmes. Nous n'aurions plus su ni pleurer ni nous abandonner. II n'y avait pas de corridor derrière. II $n^{\prime} y$ avait plus derrière nous 
que le vide le plus parfait, que le gouffre infini. Et devant? La gueule béante de Vieil Bidoune. Rien d'autre derrière que le néant absolu qui nous avait engendrés et devant nous cette gueule affamée. Mais ce désir forcené de passer au travers, de vaincre l'espace et de défoncer le temps. Nous ne pouvions plus fuir. II n'y avait nulle part où aller. Il semblait donc logique que cette terrible rage nous anime, puisque son but ultime était de nous permettre de perforer le mur temporel afin de nous faire sourdre de nouveau dans la prairie des vivants.

Quoi? Bien sûr, je mens en quelque sorte. Aucune pensée ne nous animait. Non! Nous n'étions mus que par la force vitale, que par la force initiale qui nous avait projetés vers I'avant. Le coup de jarret cosmique si vous voulez. Le rythme élémentaire mais quelquefois si trépidant.

Nous fûmes bientôt jetés vivants dans la gueule grande ouverte du monstre répugnant. II y eut comme une brisure dans nos neurones, comme une seconde d'amnésie totale dans nos têtes et, dans le noir opaque de sa bouche, nous vîmes soudainement briller, comme de lointaines étoiles, ses longs crocs malfaisants. De lointaines étoiles. Oui! Et plus de crocs carnivores. Des étoiles par milliers, dans I'infiniment lointain.

La peur nous avait dès lors quittés. Nous étions calmement installés à bord d'un vaisseau tout de verre étincellant, vêtus comme de gentils cosmonautes adolescents. Et nos rires effrontés emplissaient cristallins la Bulle au tableau de bord scintillant.

Nous avions eu raison de la deuxième épreuve. L'horreur multipliée n'était pas venue à bout de nous séparer. 
Le sort tomba sur le plus jeune le sort tomba sur le plus jeune c'est donc lui qui qui qui serait mangé c'est donc lui qui qui qui serait mangé

Désormais, les événements allaient toutefois se précipiter de plus en plus et ne nous laisser que des pauses de ressourcement toutes plus brèves et plus insuffisantes les unes que les autres.

C'est ainsi que le verre de la Bulle se mit bientôt à craqueler, à se fissurer avec des petits bruits agaçants. Nos teints roses d'enfants s'étaient veinés de zébrures vertes. Chacun, à son grand désarroi, lisait de nouveau l'angoisse épuisante sur les traits vieillis de l'autre. Toute la pureté originelle de notre romanesque scénario s'était dissoute dans un bain de LSD-25 liquide. II y avait d'énormes rides sur nos mains jointes. Nous étions déjà en passe de devenir deux nains vieux et effrayants. Et la solitude de l'espace commençait à nous peser, faisait son nid dans nos âmes.

J'ai fermé les yeux avec entêtement. J'ai plongé intérieurement jusqu'à la parfaite vision de ce champ où nous allions jouer si souvent, jusqu'à sa matérialisation presque. Puis j'ai rouvert les yeux prestement et j'ai revu Josephat Robert tel qu'il était alors. Pur et innocent. Tout à côté de moi, si près de moi, Josephat Robert enfant, mon double nécessaire. Nos petites mains charnelles l'une dans l'autre, nous filions dans I'espace, inexorablement. Le vaisseau s'était réparé.

* * * * *

L'un voulait qu'on le mit à frire I'un voulait qu'on le mit à frire I'autre voulait lait lait le fricasser l'autre voulait lait lait le fricasser

Puis, ne restèrent plus que nos deux sièges. Tout autour s'était encore métamorphosé. 
Nous fûmes dans un vaste cinéma où l'on avait maîtrisé l'art de faire paraître et ressentir profondément toutes les dimensions du réel, où l'on savait rendre compte de tout ce qui s'adresse simultanément à tous les sens. Mais plus! Quelque chose de fondamental et de très difficile à exprimer clairement. Comme une surprenante capacité de rendre perceptibles aux sens l'ensemble des codes extrêmement vastes et complexes qui régissent et expliquent la manifestation de ce que tu perçois comme le réel. Non pas du cinéma! La création instantanée d'un réel adapté aux phobies et aux phantasmes du spectateur.

Ce fut une expérience plus fascinante, plus troublante encore que notre fuite devant puis vers Vieil Bidoune et combien plus angoissante que l'apparente déconfiture de notre vaisseau de verre dans l'espace sidéral.

La projection était déjà en cours à notre arrivée. Nous étions cloués à nos fauteuils. Stupéfaits.

Mais regardant la mer entière mais regardant la mer entière il vit des flots flots flots de tous côtés il vit des flots flots flots de tous côtés

II était question de navets bleus. Elle contait à qui voulait l'entendre que, pour les bouillir, il fallait compter sur les coquilles mécaniques, le temps, un contenant, de l'eau et du feu.

Elle savait, par alchimies et soustractions, interférences et mélange des pensées, que le navet bleu était une farce. Elle riait donc. À s'en fendre les commissures jusqu'aux fesses. Elle riait. II vint. II avait un grand sourire Pepsodent de mâle fraîchement déterré. Il sortait des décombres, des éclatements suburbains récents. II avait des yeux ronds qu'il promenait sur les murs, les y roulant du bout de ses doigts secs. Quand, par 
malheur, il en laissait tomber un, il faisait un saut vers l'arrière, surpris, décontenancé, comme pour ne pas marcher dessus.

Après avoir jugé du bruit de l'oeil roulant, il s'accroupissait puis cherchait à tâtons, les quatre pattes par terre, sur le parquet clean-style. Il sentait alors sous ses doigts si sensibles l'éclat de la tuile que la très douce Helmance avait frottée et frottée... II aimait la fille de son mieux. Mais il n'avait pas conscience de l'importance du navet bleu.

Jar et moi étions subjugués.

Un jour, rentrant de Mars, les pieds encore enneigés et boueux, il glissera sur le plancher cuivré. Se cassera l'idée maîtresse. II faudra mettre le truc sous plâtre. II faudra patienter. Helmance aura de la patience pour deux. Tout d'abord, elle tentera de remplir le vide affreux par sa présence affective. Elle cuira moult navets bleus, les épilera et les servira au héros sur I'air de la délivrance. Psaume connu. Psaume historique depuis le fin fond des avenirs cocasses. Les avenirs en conserves. Les avenirs bon marché pour tous. Pour elle et pour lui, surtout pour lui.

Il faudra bien qu'un beau jour elle passe une commande de soleil entre les barreaux fixes de la cellule. Elle ploguera le fil du soleil et s'illuminera. Mais avant de se casser l'idée maîtresse, Brave Gence aura laissé tomber ses deux yeux ronds. L'idée maîtresse cassée, les deux yeux tombés, il ne verra donc pas, le pauvre homme, la douce Helmance si près de lui déguisée en soleil. Tout au plus, il sentira vaguement, mais comme de très très loin en lui que là, tout près de lui, il se produit quelque chose.

\section{MORT ÉTOUFFÉ DANS SA CELLULE!}

Puis, les chapîtres se bouleverseront. La philosophie renversée sur la nappe sera d'avant l'histoire. Le millésime même de l'histoire sera quant à lui d'avant le temps. Le temps, deve- 
nu olfactif, ne pourra plus jamais retracer les origines, bien qu'il serve à en flairer la piste. Le mal et le bien seront enfouis dans l'inconcordance. II croira peut-être en Dieu. S'il est plus sage il se croira au multiple. Ne cherchera plus d'où il était, mais plutôt où il allait quand il fût.

\section{MEURTRE SAUVAGE À CHANDLER!}

Il est venu à la cuisine, attiré par l'odeur forte des navets bleus bouillis. Il est tombé sur ses cinq sens. Helmance était nue. Délicieusement nue. Le dos certain, la croupe en S, les fesses baîllant un sourire de demains qui chantent. II se mit à genoux. L'encula. Elle commença d'apprendre à geindre. II éjacula des anges. Elle restitua Sodome.

\section{BÂTONS DE DYNAMITE CHEZ LA BELLE MANON!}

Quand elle gesticula des mains pour lui apprendre qu'elle portait un enfant, il entra dans une Sainte Colère, la bouscula, lui fit l'amour par devant le moulin d'où viens-tu bergère. II était accroché à l'idée qu'il s'était cassé l'idée maîtresse à cause d'une enculée qui lui avait fait échapper ses yeux qu'il roulait sur le mur; pusillanime distraction d'ailleurs. Qui n'en ferait pas tant pour se pénétrer de la philosophie du bagne à perpet?

\section{EXÉCUTION DE 20 VILLAGEOIS: UN "SS" PASSE AUX AVEUX!}

II y aurait de la musique. Les mains de I'homme stone montèrent la garde autour des hanches frelatées de la femme ouverte. La bouche chercha la blessure. S'y risqua et s'y complut. La femme poussa de toutes ses forces recrudescentes, tendit les jambes en un décent bandage, crispa forte- 
ment ses entrailles. Le bébé mauve, encore gluant des actes, fut alors précipité dans l'espace. Brave avait tout juste eu le temps d'amorcer un mouvement de recul biaisé pour éviter le choc. Heureusement, il eut été tué net. II faudra pêcher le mioche au filet. Pourvu qu'il ne glisse pas, par malheur ou bah, dans la voie lactée ou ailleurs.

\section{ADOLESCENT EXÉCUTÉ PAR L'ESCADRON DE LA MORT!}

D'un geste brusque, elle attrapa l'enfant volant par la chevelure. Les boucles blondes se déroulèrent quasi infiniment dans l'espace sombre. Elle parvint tout de même, tortilIonnant et tortillonnant la chevelure bouclée à tirer le gosse jusqu'à elle. L'embrassa sur la bouche. Profondément.

\section{DEUX ANGES DU MAL ATTAQUENT ET TUENT 30 TOURISTES!}

II avait grandi. II glissa ses pieds jusqu'au foyer. Planta ses talons dans les flammes sudorifères. Tourna de l'oeil à droite et sourit glacialement au jeune homme. Las de ce jeu concis, il retira ses pieds du feu, se leva et fit trois pas. II prit dans les siennes froides la main du jeune homme, qui pendait molle à I'angle du lit. II embrassa les lèvres ivres-mortes. II y eut dans sa tête l'image d'une mare stagnante troublée par la chute d'une roche plate. II tourna le cadavre à plat ventre, le couvrit une heure et quitta la pièce sans mot dire. Ils avaient été, I'un et I'autre.

\section{DOUBLE SUICIDE OU DOUBLE MEURTRE?}

La femme morte monta trois escaliers. L'homme de jeu descendit trois étages. Ce mouvement anachronique les conduisit curieusement tous les deux au sous-sol, dans une 
pièce large et unique, sans décor, sans honte. Ils ne respiraient pas. N'ayant pas d'yeux, ils se regardaient au travers des plafonds et des planchers, des sphères transversales. Pourtant, ils s'étaient vus l'un l'autre un jour.

\section{IL VIOLAIT LES FEMMES SOUS LA MENACE D'UN REVOLVER!}

II se retira. Les fesses tremblantes du garçon joli se refermaient tout doucement. Encore fumante, l'âme rose expirait des vapeurs de désirs assouvis. Ils entrelacèrent leurs membres entendus jusqu'à la vérité mon oeil. Ils s'aimaient gauches, vivaient drabe, avaient joui jaune. Ils prirent ensemble un café pâle. Se sourirent franchement caille. Le plus jeune tendit sa main gauche vers la bouche de l'autre qui la baisa du bout des lèvres. Ils moururent.

\section{UN SUICIDE PAR SEMAINE DANS LE MÉTRO!}

L'histoire racontée sera au futur. Le temps sera de la lumière-vitesse au carré, à rebrousse-poil sous la main qui flatte. $\mathrm{O}$ qu'ils avaient la fleur-de-peau entre les lèvres, miouvertes mi-closes, mi-sourire et mi-charognardes. L'un injectait à l'autre la nécessaire suie noire arrachée au néant.

\section{PROSTITUTION ET MEURTRE CHEZ LES COIFFEUSES!}

Elle était sa mère. L'enfant entra en courant, les pieds nus, ses running shoes à la main. II la vit, verte, mûrir sous ses yeux, s'étirer nonchalamment jusqu'à tout; lui-même tout entier possédé par les émanations douceâtres de son râle. II arracha d'une main soudain forte et féroce le bracelet de fer à sa cheville droite. II avait mal. 
Jar allait suffoquer.

II jeta la femme sur une peau de bête, posa sa tête sonore sur le sein gonflé doux à sa joue et enfonça sa petite verge en elle. II allait nourrir sa mère. Son pied droit saignait. Les reins de l'enfant déployèrent deux ailes roses en paravents protecteurs. Comme une onde, dans un va-et-vient soutenu, il pénétrait sa mère jusqu'au premier orgasme de la putain, peu importe le quartier, peu importe le trottoir, peu importe la saison. Très loin tout au fond, jusqu'à la première masturbation comique du Père Universel, jusqu'à l'origine des origines. II était blond, doux, bouclé, crevé comme un ballon d'hélium sans.

\section{UNE JEUNE NYMPHOMANE ÉTRANGLÉE PAR SON MARI IMPUISSANT!}

Son corps tout à coup se tordit de douleur. Comme projeté hors des sphères, le gosse disparut. II lui avait éjaculé sur la gueule. Il allait nourrir sa mère. Son pied droit ne saignait plus.

\section{BOXEUR AMATEUR BATTU À MORT À LA SORTIE D'UN BAR!}

La tante sortit. Elle marcha dans la rue grise. Rencontra un petit de sept ou huit ans. L'amena au parc. N'en tira rien. Mais Bon Dieu qu'il était beau. II eut fallu un nouveau vocabulaire. Une nouvelle échelle sensorielle eut été nécessaire pour situer sur la graphique ce désir.

\section{DOUZE ANS DE PEN POUR AVOIR BATTU À MORT SA JEUNE DANSEUSE NUE!}

Se rendant à la messe, il grelottait dans son surplis. II se 
demandait bien pourquoi la planète était sous ses pieds et non là-haut, comme la lune. Quand il entra dans la sacristie, on l'attendait déjà. Tout le peuple des nonnes, à genoux, les mains jointes, était là. L'abbé s'approcha de lui, blême et doucereux. II prit l'enfant dans ses bras maigres et l'embrassa très longuement sur la bouche. Sa longue langue mouillée fouillait la petite grotte des baumes primordiaux, s'attardant longuement au palais chatouilleux.

Le prêtre, tout ce temps-là, gardait les yeux fermés. Tout en son corps signifiait l'extase. Les nonnes, avec ardeur, bienque dans le plus parfait silence, priaient Notre Père... L'homme coucha ensuite le petit garçon sur l'autel, souleva le blanc surplis et le replia par-dessus la tête. II entreprit ensuite, dans une grande pâleur, la consécration. Les doigts habiles s'agitaient fiévreusement sur le ventre dépouillé. Le jeune sexe érigé hésitait, entre les caresses, à faire un saut, à bouger. Les cuisses blanches et frêles avaient la chair de poule, dans cette puante humidité froide des lieux saints. Au kyrie, tous les baisers collants encaissés par le petit corps, la croix noire descendit d'un coup brusque, comme autonome, se planter dans le ventre sans poil qui laissa échapper un long bruit sourd de pneu crevé et une odeur de caoutchouc brûlé.

\section{SURPRIS AVEC LA SERVANTE...}

Dans sa cellule, sous sa robe noire, elle glisse furtiyement le crucifix. Les cuisses moites gémissent toutes les plaintes que l'on refuse d'entendre. La main gauche, dans le tiroir, se crispe sur le manche de la dague. Se retire brusquement. Et la lame direct au coeur... Dans sa robe rouge, elle retourne aux catacombes. Le Christ entre l'âme et la vie lui sourit. Elle a vécu.

\section{IL TENTE D'ÉLECTROCUTER SA FEMME DANS SON BAIN!}


Quand le garçon dégela il se retira de sa mère. Laissa en elle tous les plaisirs appris par coeur. Se courba en foetus entre les cuisses grasses. Et dormit. Conté à lui-même en rêve, le rêve par le rêve. Entre ses jambes, son organe repu perdait chair. Les reins ouverts elle avait hurlé. Le vent venu juste alors avait porté aux morts ce cri de bête violée.

\section{HORRIBLE MASSACRE À L'HÔPITAL DE LA MORT!}

II approcha la petite blonde. II lui montra le vaste tableau noir, vierge pourtant (il était tout neuf). II plongea son regard brûlant dans les yeux pers de la pucelle, la prit par l'oreille et la traîna de force à la guillotine. Elle voulut bien résister, mais il tirait si fort que la fine tige qui lui tenait lieu de cou s'est étirée comme filament jusqu'à ce que la mignonne tête se trouve de I'autre bord de la lame. II contempla la scène un moment, comme de très loin en lui. Actionna. La petite blonde disparut. Il y avait perdu un bras mais, que diable, on ne réussit rien sans peine.

II approcha la petite blonde. Lui sourit. Lui parla tout doux. La cajola. La baisa dru. La jeta au fleuve.

II approcha la petite blonde. Lui parla timide et gentil. La cajola tendre. La caressa mouillé. La poussa sec sous le métro rapide.

II approcha la petite blonde. Lui dit aimablement bo'zou'. Lui baisa la main gallant. Tira six coups serrés.

\section{LE PROCÈS DU GENTIL MANIAQUE: UN DÉPUTÉ LÈCHE LE SEXE D'UNE PETITE FILLE À PEINE PUBĖRE PEN- DANT QUE SA GRANDE SOEUR LUI PASSE UN POI- GNET!}

Dans sa chambre (avec les poupées mécaniques qui pleurent, chantent, pissent et tout...) elle a quinze ans, n'est pas 
moins que très très belle, ce qui est beaucoup. Elle se fait, dans le miroir, tout au centre d'elle-même, OUI du doigt.

\section{L'HOMME-ARAIGNÉE EST ARRÊTÉ!}

Dans son fauteuil, où il allait bientôt se perdre, tout recroquevillé, rapetissant à vue d'oeil, Josephat Robert se défaisait à côté de moi.

Je l'ai donc placidement assisté dans son agonie. Intervenant juste assez pour l'aider à ne pas perdre complètement le contrôle.

Puis, prétextant pour ma propre conscience qu'ainsi je le sauverais, surtout pour ne rien perdre de sa si nécessaire présence, j'ai découpé soigneusement son cadavre rigide en tout petits morceaux que je me suis enfoncés un à un dans la gorge, incapable de les mastiquer.

Ce fut une communion totale. J'ai dépecé Josephat Robert et je l'ai avalé. Oui monsieur le juge. C'était la troisième épreuve. II n'y avait rien d'autre à faire que d'observer douloureusement. Pour survivre, nous avons fusionné.

Si cette histoire vous amuse

si cette histoire vous amuse

je vais vous la la la recomthencer

je vais vous la la la recommencer

II était un petit navire... 